\title{
A new method to quantify penile erection hardness: real-time ultrasonic shear wave elastography
}

\author{
Hao Cheng ${ }^{1,2 \#}$, Zichang Niu ${ }^{3 \#}$, Fengyue $\mathrm{Xin}^{1},{\text { Lin } \text { Yang }^{4}, \text { Litao Ruan }}^{1 \wedge}$ \\ ${ }^{1}$ Department of Ultrasonography, First Affiliated Hospital of Xi'an Jiaotong University, Xi'an, China; ${ }^{2}$ Department of Ultrasonography, Shaanxi \\ Province Cancer Hospital, Xi'an, China; ${ }^{3}$ Department of Ultrasonography Special Diagnostic, Air Force 986 Hospital, PLA, Xi'an, China; \\ ${ }^{4}$ Department of Urology, First Affiliated Hospital of Xi'an Jiaotong University, Xi'an, China \\ Contributions: (I) Conception and design: L Ruan, L Yang; (II) Administrative support: L Ruan; (III) Provision of study materials or patients: L Ruan,
} L Yang ; (IV) Collection and assembly of data: H Cheng, Z Niu, F Xin; (V) Data analysis and interpretation: H Cheng, F Xin; (VI) Manuscript writing: All authors; (VII) Final approval of manuscript: All authors.

"These authors contributed equally to this work.

Correspondence to: Litao Ruan, MD, PhD. Department of Ultrasonography, First Affiliated Hospital of Xi'an Jiaotong University, Xi'an 710061, China. Email: ruanlitao@163.com; Lin Yang, MD, PhD. Department of Urology, First Affiliated Hospital of Xi'an Jiaotong University, Xi'an 710061, China. Email: docyanglin@126.com.

Background: An important indicator of penile erectile function is erection hardness (EH), which is currently evaluated by the semi-quantitative erectile hardness score (EHS). EH increases continuously during the course of an erection, so although it is statistically a continuous variable, the EHS is a grade variable. We propose a new method for real-time quantitative measurement of penile $\mathrm{EH}$ using ultrasonic shear wave elastography (SWE).

Methods: The study group comprised 40 patients with erectile dysfunction (ED) and 20 normal controls who all underwent real-time SWE to measure tissue stiffness (Young's modulus, YM) of the penile corpus cavernosum and tunica albuginea during erection, at rest and at different $\mathrm{EH}$ grades induced by intracavernosal injection (ICI) of prostaglandin. The examiner gently placed the high frequency probe on the ventral penis and got the two-dimensional longitudinal US image of penis, then switched to SWE mode, the appropriate region of interest (ROI) was selected, then a 3-5 $\mathrm{mm}$ circle ("Q-box") was automatically set to by machine to measure the SWE of the corpus cavernosum and tunica albuginea on the left side.

Results: In both the ED patients and normal controls, YM slightly decreased in the corpus cavernosum during erection but the stiffness of tunica albuginea increased significantly with increasing EH (resting: $21.66 \pm 4.21 \mathrm{kPa}$, grades $1-4: 32.61 \pm 4.27,54.86 \pm 8.69,128.02 \pm 20.66$, and $223.23 \pm 23.61 \mathrm{kPa}$, respectively). Because EH grades 1 and 2 clinically predicted failure to complete sexual intercourse, and EH grades 3 and 4 predicted ability, the cutoff point for the YM of the tunica albuginea to evaluate whether sexual intercourse could be completed was $81.60 \mathrm{kPa}$.

Conclusions: Our data demonstrated that tunica albuginea stiffness, not corpus cavernosum stiffness, provided a good clinical imaging index indicator for evaluating penile EH. The tunica albuginea stiffness changed continuously during penile erection, which could be measured quantitatively by SWE. Compared with the EHS, measuring the YM of the penile tunica albuginea using SWE is a new and objective technique for quantitatively assessing EH. Because of its objective and quantifiable characteristics, measuring YM enables more accurate evaluation of the effect on EH of various treatments for ED.

Keywords: Corpus cavernosum; erectile dysfunction (ED); erection hardness; tunica albuginea; ultrasound shear wave elastography (ultrasound SWE)

Submitted Jun 28, 2020. Accepted for publication Aug 05, 2020.

doi: $10.21037 / \mathrm{tau}-20-1096$

View this article at: http://dx.doi.org/10.21037/tau-20-1096

$\wedge$ ORCID: 0000-0002-0657-414X. 


\section{Introduction}

Erectile dysfunction (ED) is one of the most common male sexual dysfunctions $(1,2)$, with the Massachusetts Male Aging Study reporting an overall prevalence of $52 \%$ in noninstitutionalized men aged 40-70 years (3). A global survey of 29 countries found that the incidence rate was about $13-28 \%$ (4). It has become a major problem worldwide, with approximately 150 million men afflicted with various degrees of ED, and the number of patients is predicted to double by 2025 (5). The cause of ED may be organic (neurogenic, hormonal, vasculogenic, or drug induced), psychogenic (anxiety, depression, relationship distress), or mixed. It is recognized that ED treatment should be approached in a multidisciplinary fashion including urology, andrology, endocrinology, and internal medicine.

Erection hardness $(\mathrm{EH})$ is an important indicator of ED and is typically assessed using a tactile tool (6). The close and direct relationship between erection hardness and successful sexual intercourse supports the broader use of the erectile hardness score (EHS) in clinical practice. EHS (7-9) is a simple, validated, semi-quantitative, self-reported tool that scores $\mathrm{EH}$ on a 4-point scale: 0, penis does not enlarge; 1 , penis is larger but not hard; 2 , penis is hard but not hard enough for penetration; 3 , penis is hard enough for penetration but not completely hard; 4: penis is completely hard and fully rigid. The EHS depends on the patient's subjective feelings, but because $\mathrm{EH}$ increases continuously during the course of an erection, it is statistically a continuous variable, whereas the EHS is a grade variable. Thus, a quantitative measurement of $\mathrm{EH}$ would be more objective and accurate. In the clinic practice, there was no other method to measure the EH except EHS.

Real-time shear wave elastography (SWE) is a type of ultrasonography (US) elastography that uses shear waves to assess tissue elasticity and display it in a quantitative manner. Unlike acoustic compressive waves, which spread in the same direction as the particle compression, the propagation of shear waves proceeds orthogonal to the stimulated displacement. Shear waves can result from an acoustic radiation force, mechanical punch, or external sources. The SWE probe is able to transmit and capture shear waves, which are used to calculate Young's modulus (YM), with a higher YM value indicating greater stiffness. Tissue stiffness is displayed in real-time with color-coding technology (10-13). SWE has been used effectively in the diagnosis and differential diagnosis of prostatic (10), hepatic (11), thyroid (12), and breast disease (13). Its application for ED is limited to only a few studies that have not taken into account the quantitative aspects of $\mathrm{EH}$ $(14,15)$. We know that $\mathrm{EH}$ increases during an erection, and that SWE examination could be used to measure the stiffness, but we do not know whether SWE can be used to quantitatively measure $\mathrm{EH}$. In this study, we explored using this method to quantitatively evaluate penile EH in ED patients and normal controls.

We present the following article in accordance with the MDAR reporting checklist (available at http://dx.doi. org/10.21037/tau-20-1096).

\section{Methods}

\section{Patient selection}

This study was approved by the Ethics Committee of the First Affiliated Hospital of Xi'an Jiao University (XJTU1AF-CRF-2016-015). The clinical trial was registered online (registration no. ChiCTR2000029370). Prior to study enrollment, all subjects agreed to and signed an informed consent form. All procedures performed in this study involving human participants were in accordance with the Declaration of Helsinki (as revised in 2013). The study group comprised $40 \mathrm{ED}$ patients (age range 23-58 years, mean $35.1 \pm 10.8$ years) and 20 healthy age-matched normal controls (age range $25-58$ years, mean $34.9 \pm 11.2$ years). All subjects were married or had regular sex partners. ED was diagnosed using the International Index of Erectile Function Questionnaire (IIEF-5) (16), utilizing nocturnal penile tumescence (NPT) testing (17). Patients had an average 12.5 -month history of ED. We excluded any subjects with a history of pelvic or penile trauma or surgery, penile curvature, Peyronie's disease, congenital penile malformation or other structural abnormality. the other ED patients were taken into our study. This was confirmed by an in-depth medical history, body examination, or twodimensional US.

\section{Instruments and technique}

An Aixplorer US system (Aixplorer, Supersonic Imagine, Aix-en-Provence, France) with a Superlinear SL15-4 probe [frequency 7.5-15 MHz, Mechanical Index (MI): 1.5, Thermal Index of Soft Tissue (TIS): 1.2] was utilized. We performed examinations in a quiet, comfortable room with only the patient and examiner present. The patient was lay supine on the examination bed until he was relaxed and 
breathing evenly. Alprostadil El (prostaglandin E1, Taide Pharmaceutical Co., Ltd, Beijing, China) $5 \mu \mathrm{g}$ was injected into the corpus cavernosum to induce penile erection. An index finger was placed on the glans penis to gently draw the dorsal penis to the abdominal wall, applying as little pressure as possible. The examiner gently placed the probe on the ventral penis and used the two-dimensional US mode to scan the entire penis, freezing the image with the corpus cavernosum's organizational structure clearly shown (14). Gel was reapplied between the probe and the ventral penis and the pressure from the probe's weight and the examiner's hand on the penis was minimized. The imaging equipment was switched to SWE mode, with kiloPascals $(\mathrm{kPa})$ as the measurement unit. The image was optimized and adjusted to a standard setting (e.g., frequency range of 7.5-15 MHz, MI: 1.5 TIS: 1.2). The corpus cavernosum and tunica albuginea were displayed in longitudinal section, and three images were obtained for the bilateral corpus cavernosa and tunica albuginea tissues in real time. Another intracavernosal injection (ICI) (18) of $5 \mu \mathrm{g}$ of Alprostadil El was given to induce penile erection and twodimensional US and SWE images of the different grades of EH were obtained. In order to keep the high repeatability, all operators have more than 10 years ultrasonography experience.

\section{SWE measurement}

The appropriate region of interest (ROI) was selected for sampling frame size based on penile length. As the ROI sampling box reached its filling point with the uniform blue color, tissue stiffness was determined. At this point, a 3-5-mm circle ("Q-box"), which was automatically set by machine was selected to measure the SWE of the corpus cavernosum and tunica albuginea on the left side. The average of three points was taken and then the right side was measured under the same conditions.

\section{Statistical analysis}

SAS 9.0 software (SAS, Chicago, IL, USA) was used for statistical analyses. Quantitative data are expressed as mean \pm standard deviation (SD). A paired $t$-test was used to compare the right and left tunica albuginea and corpus cavernosum tissues at different EH. A P value $<0.05$ was considered statistically significant. Because EH grades 1 and 2 clinically prognosticate failure to complete sexual intercourse, while grades 3 and 4 are sufficient for completing sexual intercourse (7), we drew receiveroperating characteristic (ROC) curves for grades 1 and 2 and grades 3 and $4 \mathrm{EH}$ of the tunica albuginea and corpus cavernosum respectively. The Mann-Whitney test was used to compare the area under curve (AUC) of the ROC, and $\mathrm{P}<0.05$ was considered to be statistically significant. Diagnostic cutoff points between grades 1 and 2 and grades 3 and $4 \mathrm{EH}$ were also determined where indicated.

\section{Results}

\section{YM of tunica albuginea and corpus cavernosum in the left and right penis}

Among the $40 \mathrm{ED}$ patients enrolled, some were unable to achieve EH grade 2, 3, or 4 after ICI; in some patients, EH increased very slowly, which provided a special opportunity for evaluation because there was sufficient time to take SWE measurements of the stages of EH to obtain more accurate results. Even for an experienced US operator, there was approximately $20-40 \mathrm{~s}$ to capture an appropriate SWE image for measurement. In some subjects, particularly the healthy controls, the penis erected very quickly, and in several it took less than 2 minutes for EHS to increase from grade 1 to grade 4 after ICI. Therefore, SWE testing could not be completed for all grades of $\mathrm{EH}$ on both sides of the corpus cavernosum and tunica albuginea, resulting in significantly different numbers of cases of hardness for all grades (Table 1).

Thus, to observe the differences in the stiffness values of the corpus cavernosum and albuginea tunica during erection, we compared the YM values of the right and left sides of the penis, the results of which are shown in Table 1 . We did not observe any significant differences between the two sides, suggesting that a unilateral penile elasticity test was sufficient to reflect whole penis status during erection.

\section{$Y M$ values of the left albuginea tunica and corpus cavernosum according to EHS grade in ED patients and normal controls}

YM of the corpus cavernosum decreased slightly after erection, whereas it increased significantly in the tunica albuginea during erection. As shown in Figure 1, in the resting state SWE showed both the corpus cavernosum and tunica albuginea as dark blue. The color of the cavernosum did not changed significantly as EH increased, whereas the color change in the tunica albuginea was significant 
Table 1 Tissue stiffness values (Young's modulus, YM) of the left and right corpus cavernosum and tunica albuginea at different degrees of erectile hardness

\begin{tabular}{|c|c|c|c|c|c|c|}
\hline $\begin{array}{l}\text { Erectile hardness } \\
{\left[\# \text { cases }^{\star}\right]}\end{array}$ & \multicolumn{3}{|c|}{ YM of corpus cavernosum ( $\mathrm{kPa})$} & \multicolumn{3}{|c|}{ YM of tunica albuginea (kPa) } \\
\hline Resting [60] & $11.16 \pm 3.16$ & $11.05 \pm 2.97$ & 0.961 & $21.66 \pm 4.21$ & $21.97 \pm 4.51$ & 0.988 \\
\hline Grade 1 [16] & $10.22 \pm 2.23$ & $10.17 \pm 1.98$ & 1.000 & $32.61 \pm 4.27$ & $32.79 \pm 4.71$ & 0.901 \\
\hline Grade 2 [43] & $9.28 \pm 1.21$ & $9.23 \pm 1.76$ & 0.955 & $54.86 \pm 8.69$ & $54.98 \pm 9.09$ & 0.972 \\
\hline Grade 4 [37] & $9.28 \pm 1.57$ & $9.26 \pm 1.39$ & 0.968 & $223.23 \pm 23.61$ & $223.99 \pm 26.73$ & 0.990 \\
\hline
\end{tabular}

Results are shown as mean \pm standard deviation (SD). *, some erectile dysfunction (ED) patients were unable to achieve EH grade 2 , 3 , or 4, and some normal controls achieved erection very quickly after drug injection; therefore, the number of cases varies for the different erection hardness $(\mathrm{EH})$ grades.
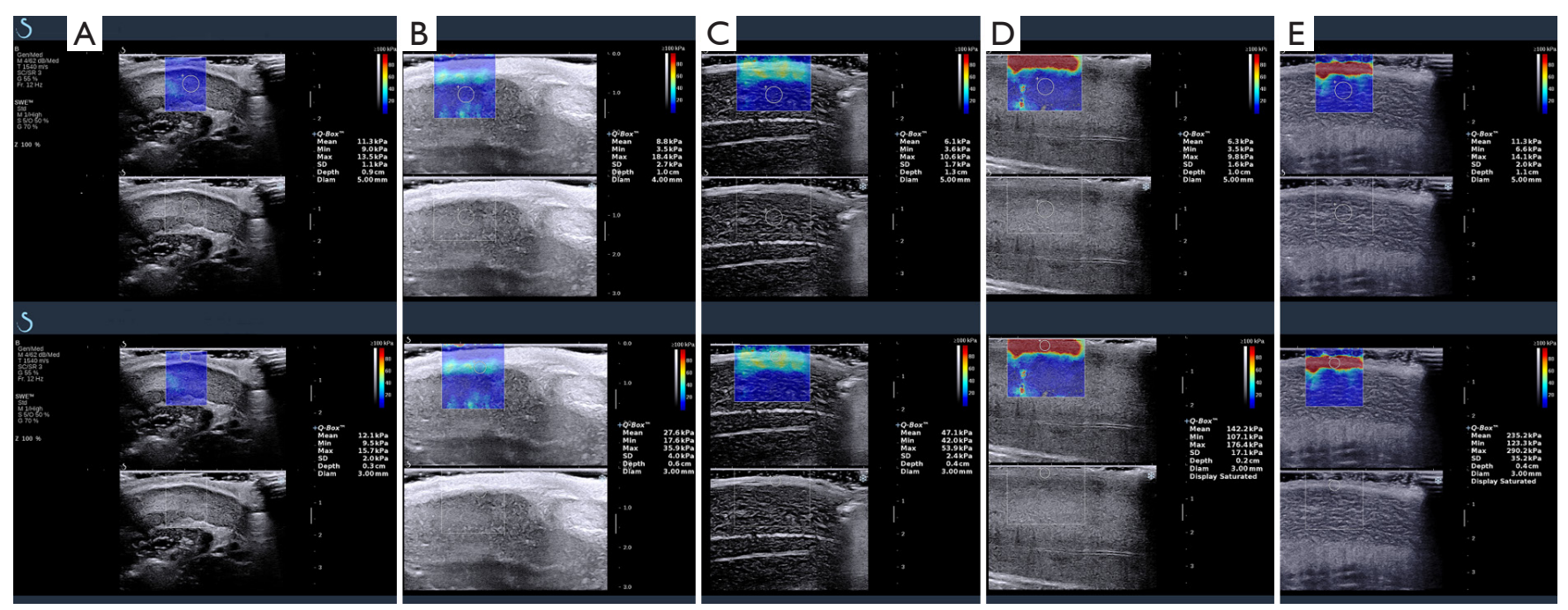

Figure 1 Real-time shear wave elastography of corpus cavernosum and albuginea tissues for different penile erection grades. (A) Up: Cavernosum at Resting state hardness (mean YM =11.3 kPa); Down: Albuginea at Resting state hardness (mean YM =12.1 kPa). (B) Up: Cavernosum at Grade 1 hardness (mean YM =8.8 kPa); Down: Albuginea at Grade 1 hardness (mean YM =27.6 kPa); (C) Up: Cavernosum at Grade 2 hardness (mean YM =6.1 kPa); Down: Albuginea at Grade 2 hardness (mean YM =47.1 kPa); (D) Up: Cavernosum at Grade 3 hardness (mean YM=6.3 kPa); Down: Albuginea at Grade 3 hardness (mean YM =142.2 kPa); (E) Up: Cavernosum at Grade 4 hardness (mean $\mathrm{YM}=11.3 \mathrm{kPa}$ ); Down: Albuginea at Grade 4 hardness (mean YM=235.2 kPa). YM, Young's modulus.

(0, dark blue; 1 , light blue; 2, blue-yellow; 3 , red; 4, dark red). Different colors indicated different stiffness; the color change of the tunica albuginea from blue to red indicated increased stiffness.

SWE could quantify the stiffness in the corpus cavernosum during penile erection, and the $\mathrm{YM}$ was as follows: resting: $11.16 \pm 3.16 \mathrm{kPa}$; grade $1: 10.22 \pm 2.23 \mathrm{kPa}$; grade 2: $9.28 \pm 1.21 \mathrm{kPa}$; grade $3: 9.26 \pm 1.71 \mathrm{kPa}$; and grade 4: $9.28 \pm 1.57 \mathrm{kPa}$. Although the YM values decreased slightly for the cavernosum tissue between the resting state and $\mathrm{EH}$ grades $1-4$, there was no significant difference. In addition, there were no significant differences between grades 1, 2, 3, and 4, no significant differences between key grades 2, 3, and 4, and no differences between grades 3 and 4 , which suggested that the corpus cavernosum tissue stiffness could not be used to quantitatively reflect penile $\mathrm{EH}$. In the tunica albuginea, the YM increased significantly during penile erection as follows: resting: $21.66 \pm 4.21 \mathrm{kPa}$; 
Table 2 Tissue stiffness values (Young's modulus, YM) of the left corpus cavernosum and tunica albuginea in patients with erectile dysfunction (ED) and normal controls (NCs) at different degrees of erectile hardness

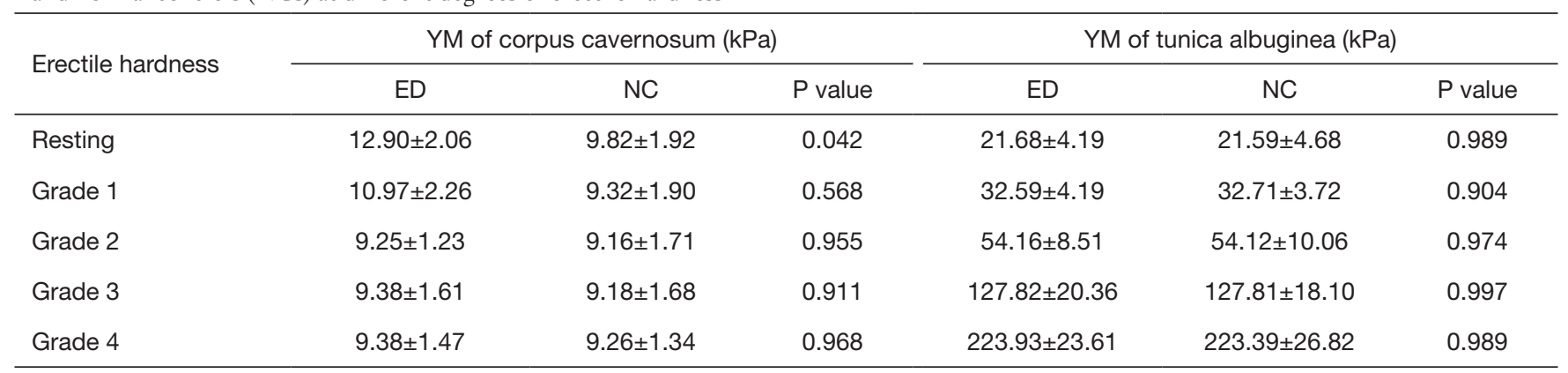

Results are mean \pm standard deviation (SD). There were no significant differences between the ED patients and NCs under SWE measurement except that the YM of the corpus cavernosum was lower in the NCs than in ED patients.

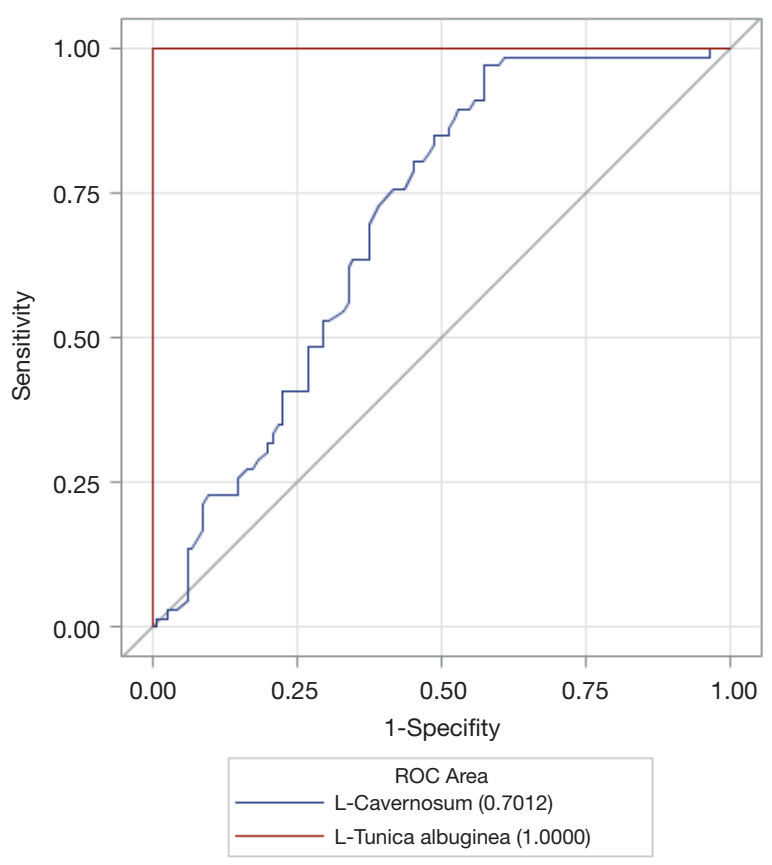

Figure 2 Receiver operating characteristics curve of Young's modulus of penile albuginea and corpus cavernosum on the left side.

grade $1: 32.61 \pm 4.27 \mathrm{kPa}$; grade $2: 54.86 \pm 8.69 \mathrm{kPa}$; grade 3: $128.02 \pm 20.66 \mathrm{kPa}$; and grade $4: 223.23 \pm 23.61 \mathrm{kPa}$. Therefore, our data demonstrated that tunica albuginea stiffness, but not that of the corpus cavernosum, is a reasonable clinical imaging index of penile $\mathrm{EH}$ from a quantitative standpoint. Because of the incomplete data sets, an analysis of variance (ANOVA) for repeated observation data at different penile EH grades could not be undertaken, and only paired comparisons were made.
Table 3 Area under the receiver operating characteristics curve (AUC) between corpus cavernosum and tunica albuginea for penile erection hardness.

\begin{tabular}{lccccc}
\hline & AUC & $\begin{array}{c}\text { Standard } \\
\text { error }\end{array}$ & \multicolumn{2}{c}{$\begin{array}{c}\text { 95\% credibility } \\
\text { interval }\end{array}$} \\
\cline { 1 - 4 } Corpus cavernosum & 0.7012 & 0.0382 & 0.6263 & 0.7761 \\
Tunica albuginea & 1.0000 & 0.0000 & 1.0000 & 1.0000 \\
\hline$\chi^{2}$ test $=61.10, \mathrm{P}<0.0001$ & & & &
\end{tabular}

The same results were obtained in both ED patients and normal controls except that the YM of the corpus cavernosum in normal controls was lower than that in ED patients at rest (Table 2).

\section{ROC curve of YM of penile tunica albuginea and corpus cavernosum on the left side}

Figure 2 shows the ROC curve of the YM of the left-sided tunica albuginea and corpus cavernosum. As shown in Table 3, the AUC of the tunica albuginea was significantly higher than that of the corpus cavernosum, which suggested that YM values of penile tunica albuginea could be used to evaluate EH. Because EH grades 1 and 2 clinically predicted failure to complete sexual intercourse, and EH grades 3 and 4 predicted ability to complete sexual intercourse, the optimal cutoff point for the YM of the tunica albuginea to evaluate whether sexual intercourse could be completed was $81.60 \mathrm{kPa}$.

\section{Discussion}

ED is one of the most common types of male sexual 
dysfunction $(1,2)$, and currently the EHS is the usual measurement tool for clinical evaluation of penile $\mathrm{EH}(7,19)$. However, although the EHS is simple and convenient, it is subjective, and semiquantitative in nature. To the best of our knowledge, an objective quantitative method of evaluating penile $\mathrm{EH}$ does not exist to date.

Real-time SWE is a non-invasive US inspection technology. It transmits a safe, acoustic radiation pulse through the probe to the tissue to induce excitation (10-13). $\mathrm{YM}$ is a measure of tissue elasticity, and the relation of $\mathrm{YM}$ and the shear wave is $E=3 P c^{2}$, where $E$ is $\mathrm{YM}, c$ is the propagation velocity of the shear wave, and $\mathrm{P}$ is the tissue density; a large YM value indicates greater stiffness. US technology can show tissue stiffness through elastography in real time using color coding $(20,21)$. Currently, SWE is used for the diagnosis and differential diagnosis of various diseases, most notably, breast and thyroid nodules $(12,13)$, because the YM for malignant lesions is significantly high as compared with benign lesions (10-13).

Zhang et al. used SWE to evaluate penile cavernosum tissue in 40 healthy volunteers (aged 19-81 years), and found that the YM values of the cavernosum tissues in young males were significantly higher than in older males $(\mathrm{P}<0.05)$, which positively correlated with the patient's testosterone level (14). Inspired by this application of SWE technology, we evaluated whether SWE technology could be used to quantitatively measure penile $\mathrm{EH}$ dynamically.

It is widely accepted that $\mathrm{EH}$ increase is based on tunica stiffness, not on cavernosum stiffness (22). However, by using SWE, we observed for the first time that the stiffness of both the tunica and cavernosum changed during penile erection. Although the cavernosum stiffness remained almost unchanged (the SWE color-coding remained blue from grade 1 to grade 4), tunica stiffness changed significantly (color change from blue to yellow, to red, and dark red). The color difference was then translated into a numerical unit, known as YM.

Our study findings suggested a method of objectively and accurately evaluate $\mathrm{EH}$ changes before and after different treatments for ED. Based on the EHS, if EH increased from grade 1 to grade 2, just one point change of the EHS scale was evaluated. However, based on our SWE data, the YM of the tunica albuginea grew continuously from 32.6 to $54.9 \mathrm{kPa}$. Even within the same grade of $\mathrm{EH}$, the YM could be significantly different. For example, in grade 4, the YM may change from 198 to $250 \mathrm{kPa}$. If we evaluated the effect of treatments on $\mathrm{EH}$, the EHS would not change within grade 4, but with SWE measurement, the treatment may have caused a change from 200 to $240 \mathrm{kPa}$, which would be a significant effect on $\mathrm{EH}$. More interestingly, if penile $\mathrm{EH}$ is between grade 3 and grade 4, the EHS cannot describe the EH, whereas SWE measurement, because of its quantitative characteristics, can accurately describe EH at any point of $\mathrm{EH}$ between grades 3 and 4 . This quantitative assessment will be especially useful for evaluating the effect of drugs and other treatments on $\mathrm{EH}$.

In addition to the corpus cavernosum, the tunica albuginea also plays an important role in the erection process (22). During penile erection, the tunica albuginea is under pressure. With an increase in $\mathrm{EH}$, tunica albuginea tension simultaneously increases, its deformation capacity correspondingly decreases, and EH increases. Our data support the view that, with an increase in penile $\mathrm{EH}$, the YM values of the tunica albuginea significantly increase, which occurred in both ED patients and healthy controls.

The comparison of the AUC of the ROC curves for the $\mathrm{YM}$ of the tunica and cavernosum tissues also showed that the AUC for the tunica albuginea ROC curve was significantly better for describing $\mathrm{EH}$ than that of the corpus cavernosum $\left(\chi^{2}=61.10, \mathrm{P}<0.0001\right)$. Clinically, penile $\mathrm{EH}$ that reaches grade 3 means that the penis can be inserted into the vagina during intercourse $(7,19)$. Therefore, we classified $\mathrm{EH}$ grades 1 and 2 as one group and $\mathrm{EH}$ grades 3 and 4 as another group to draw the ROC curves and obtain the optimal diagnostic cutoff point. This grouping showed that when the YM for the tunica albuginea was $>81.60 \mathrm{kPa}, \mathrm{EH}$ reached $\geq$ grade 3 , permitting successful sexual intercourse. This cutoff point provided an important quantifiable indicator for the clinical diagnosis and treatment of ED.

\section{Study limitations}

First, SWE measurements require specialized US equipment and a quiet examination room; it is less convenient than the EHS, and at this point, it can be used only for research purposes. In addition, erection induced by an ICI does not represent a normal physiological erection, which may have introduced a bias in the cutoff value for the study. Finally, we could not obtain stiffness values of $\mathrm{EH}$ for all four grades in all subjects; thus, our data collection was limited. Therefore, ANOVA for repeated observation data could not be used, which affects the scientific value of the results. In a subsequent study, we plan to expand the sample size to make up for this shortcoming. The area of the measured ROI was circular with a minimum diameter of $3 \mathrm{~mm}$, 
so measurement of tunica albuginea stiffness included some penile skin. It did not affect the clinical application of this method.

\section{Conclusions}

We determined that a quantitative evaluation of penile $\mathrm{EH}$ could be made by measuring the stiffness of the penile tunica albuginea instead of the corpus cavernosum while incorporating the YM with SWE. SWE is a new, noninvasive, quantitative method that can be used to evaluate penile EH. Because of its objectivity and quantitative characteristics, YM will be helpful for better evaluating the effect of various treatments for ED.

\section{Acknowledgments}

Dr. Jun Lv kindly performed statistical analyses for this manuscript.

Funding: This study received funding from the Key Clinical Projects of the First Affiliated Hospital of Xi'an Jiaotong University XJTU1AF-CRF-2016-015 and XJTU1AF2019LSL-047.

\section{Footnote}

Reporting Checklist: The authors have completed the MDAR reporting checklist. Available at http://dx.doi.org/10.21037/ tau-20-1096

Data Sharing Statement: Available at http://dx.doi. org/10.21037/tau-20-1096

Conflicts of Interest: All authors have completed the ICMJE uniform disclosure form (available at http://dx.doi. org/10.21037/tau-20-1096). The authors have no conflicts of interest to declare.

Ethical Statement: The authors are accountable for all aspects of the work in ensuring that questions related to the accuracy or integrity of any part of the work are appropriately investigated and resolved. This study was approved by the Ethics Committee of the First Affiliated Hospital of Xi'an Jiao University (XJTU1AFCRF-2016-015). The clinical trial was registered online (registration no. ChiCTR2000029370). Prior to study enrollment, all subjects agreed to and signed an informed consent form. All procedures performed in this study involving human participants were in accordance with the Declaration of Helsinki (as revised in 2013).

Open Access Statement: This is an Open Access article distributed in accordance with the Creative Commons Attribution-NonCommercial-NoDerivs 4.0 International License (CC BY-NC-ND 4.0), which permits the noncommercial replication and distribution of the article with the strict proviso that no changes or edits are made and the original work is properly cited (including links to both the formal publication through the relevant DOI and the license). See: https://creativecommons.org/licenses/by-nc-nd/4.0/.

\section{References}

1. EAU Guidelines. Edn. presented at the EAU Annual Congress Amsterdam 2020. ISBN 978-94-92671-07-3.

2. Chung E. Stem cell therapy in diabetic men with erectile dysfunction: a step closer to safe and effective regenerative technology. Ann Transl Med 2019;7:S40.

3. Feldman HA, Goldstein I, Hatzichristou DG, et al. Impotence and its medical and psychosocial correlates: results of the Massachusetts Male Aging Study. J Urol 1994;151:54-61.

4. Laumann EO, Nicolosi A, Glasser DB, et al. Sexual problems among women and men aged 40-80y: prevalence and correlates identified in the Global Study of Sexual Attitudes and Behaviors. Int J Impot Res 200;17:39-57.

5. Kalsi JS, Kell PD. Update on oral treatments for male erectile dysfunction. J Eur Acad Dermatol Venereol 2004;18:267-74.

6. Limoncin E, Gravina GL, Lotti F, et al. The Masturbation Erection Index (MEI): validation of a new psychometric tool, derived from the six-item version of the International Index of Erectile Function (IIEF-6) and from the Erection Hardness Score (EHS), for measuring erectile function during masturbation. BJU Int 2019;123:530-7

7. Mulhall JP, Goldstein I, Bushmakin AG, et al. Validation of the erection hardness score. J Sex Med 2007;4:1626-34.

8. Goldstein I, Lue TF, Padma-Nathan H, et al. Oral sildenafil in the treatment of erectile dysfunction. Sildenafil study group. N Engl J Med 1998;338:1397-404.

9. Goldstein I, Mulhall JP, Bushmakin AG, et al. The erection hardness score and its relationship to successful sexual intercourse. J Sex Med 2008;5:2374-80.

10. Correas JM, Tissier AM, Khairoune A, et al. Prostate cancer: diagnostic performance of real-time shear-wave elastography. Radiology 2015;275:280-9. 
11. Barr RG. Shear wave liver elastography. Abdom Radiol (NY) 2018;43:800-7.

12. Hu X, Liu Y, Qian L. Diagnostic potential of real-time elastography (RTE) and shear wave elastography (SWE) to differentiate benign and malignant thyroid nodules: A systematic review and meta-analysis. Medicine (Baltimore) 2017;96:e8282.

13. Athanasiou A, Tardivon A, Tanter M, et al. Breast lesions: quantitative elastography with supersonic shear imaging-preliminary results. Radiology 2010;256:297-303.

14. Zhang JJ, Qiao XH, Gao F, et al. A new method of measuring the stiffness of corpus cavernosum penis with shearwave $^{\text {TM }}$ elastography. Br J Radiol 2015;88:20140671.

15. Qiao XH, Zhang JJ, Gao F, et al. An experimental study: evaluating the tissue structure of penis with 2D-ShearWave ${ }^{\mathrm{TM}}$ Elastography. Int J Impot Res 2017;29:12-6.

16. Rosen RC, Riley A, Wagner G, et al. The international index of erectile function (IIEF): a multidimensional scale for assessment of erectile dysfunction. Urology 1997;49:822-30.

Cite this article as: Cheng H, Niu Z, Xin F, Yang L, Ruan L. A new method to quantify penile erection hardness: realtime ultrasonic shear wave elastography. Transl Androl Urol 2020;9(4):1735-1742. doi: 10.21037/tau-20-1096
17. Matsuda Y, Hisasue S, Kumamoto Y, et al. Correlation between erection hardness score and nocturnal penile tumescence measurement. J Sex Med 2014;11:2272-6.

18. Sikka SC, Hellstrom WJ, Brock GMorales AM. Standardization of vascular assessment of erectile dysfunction: standard operating procedures for duplex ultrasound. J Sex Med 2013;10:120-9.

19. Mulhall JP, Levine LA, Jünemann KP. Erection hardness: a unifying factor for defining response in the treatment of erectile dysfunction. Urology 2006;68:17-25.

20. Sarvazyan AP, Rudenko OV, Swanson SD, et al. Shear wave elasticity imaging: a new ultrasonic technology of medical diagnostics. Ultrasound Med Biol 1998;24:1419-35.

21. Bercoff J, Tanter M, Fink M. Supersonic Shear Imaging: A New Technique for Soft Tissue Elasticity Mapping. IEEE Trans Ultrason Ferroelectr Freq Control 2004;51:396-409

22. Andersson KE, Wagner G. Physiology of penile erection. Physiol Rev 1995;75:191-236.

(English Language Editor: K. Brown) 